\title{
Possibilistic Clustering Algorithm Incorporating Grey-Level Histogram and Spatial Information for Image Segmentation
}

\author{
Jing Yu, Yujun Kuang, and Xinchuan Fu
}

Mobilelink, UESTC, Chengdu, China

\begin{abstract}
Image segmentation is a process of segmenting an image into non-intersecting regions containing homogeneous pixels that are inhomogeneous with those in other adjacent regions. In this paper, a possibilistic clustering algorithm incorporating grey-level histogram and spatial information (PCA_HS) for image segmentation is proposed. The grey-level histogram speeds up the algorithm and the spatial information enhances its robustness to noise and outliers. To assess the proposed algorithm, four widely used validity indexes are computed and discussed. As the experimental quantitative and qualitative results on real images with and without noise show, PCA_HS can preserve the homogeneity and integrality of the regions and hence is more effective and efficient than traditional PCA.
\end{abstract}

\section{Introduction}

Image segmentation plays an important role in a variety of applications such as object recognition, image understanding and computer vision. The task of image segmentation is to partition an image into a number of non-overlapping regions with homogeneous characteristics while the union of any two spatially adjacent regions is inhomogeneous. To date, many researchers have proposed various segmentation algorithms [1-4]. Among these methods, fuzzy clustering is one of the most popular types of methods for image segmentation, which introduces fuzziness for the belonging-ness of each image pixel and thus can retain more information from the original image [5].

Fuzzy C-Means (FCM) algorithm was first introduced by Dunn and later extended by Bezdek, which now is one of the most widely used fuzzy clustering algorithms in image segmentation [6]. However, since the memberships of FCM are relative values depending on each other, it is sensitive to noise, outliers and other imaging artifacts. To overcome this weakness and produce memberships that have a more reasonable explanation of the degree of belonging for the data, Krishnapuram and Keller [7, 8] proposed a possibilistic approach to clustering (PCM1) and later a parallel one (PCM2) using a possibilistic type of membership functions. These algorithms with possibilistic memberships were proved to be more robust to noise and outliers than FCM. However, M. Barni, et al. [9] has proved that PCM1 to be sensitive to the initial prototypes since no link exists among clusters, which tends to produce coincident clusters. Furthermore, the performance of PCM1 heavily depends on the parameter $\eta_{i}$ and the fuzzifier $m[8,9]$. PCM2, on the other hand, is also sensitive to parameter $\eta_{i}$. In addition, it omits the fuzzifier $m$ and thus cannot flexibly adjust any parameter to get reasonable clustering results when coincident clusters occur. Based on the FCM objective function, the partition coefficient (PC) and partition entropy (PE) validity indexes, Yang and $\mathrm{Wu}[10]$ proposed another possibilistic clustering algorithm (PCA). In fact, PCA is similar to PCM2, except for the inclusion of the fuzzifier $m$ and the cluster number $c$ into membership functions, and the replacement of $\eta_{i}$ with a relatively constant parameter $\beta$. However, as the experimental results show, the direct application of PCA on an image will segment out many isolated pixels, which is undesirable.

An image has two important features that are useful for segmentation. One is the grey-level histogram, which can extremely decrease the spatial and computational complexity. To utilize this feature, $\mathrm{Xu}$ [11] proposed the Genetic Fuzzy C-Means Algorithm. Despite its time efficiency, this algorithm is sensitive to noise and outliers. Another important feature is the spatial information, which can enhance the robustness to noise and outliers. Based on FCM or PCM, Many improved clustering methods have been proposed by incorporating spatial information into the membership or objective functions [5, $6,12,13]$. Chuang et al. [12] proposed sFCMp,q, which defined the spatial functions and incorporated them into the membership functions of FCM. The drawback of $\mathrm{sFCMp}, \mathrm{q}$ is that the membership functions are computed in each iteration step, which is very time-consuming. Zuo [13] improved PCM1 by updating the memberships to the 
geometric mean of its neighbouring memberships in each iteration step, which is also time-consuming. Hence, in this study, our motivation is to take advantage of the greylevel histogram to speed up the algorithm and make use of the spatial information to enhance its robustness to noise and outliers as well.

The remainder of this paper is organized as follows. In the next section, PCA is simply reviewed, and then the proposed algorithm is described in detail, followed by a brief introduction of the four validity indexes generalized in [10]. In Section III, we use some natural images to compare the efficiency and robustness between PCA and PCA_HS. The validity indexes are computed and discussed after each segmentation. Finally, conclusions are drawn in section IV.

\section{Methodologies}

\subsection{PCA}

Based on the FCM objective function, the partition coefficient (PC) and partition entropy (PE) validity indexes, Yang and $\mathrm{Wu}[10]$ proposed another possibilistic clustering algorithm (PCA). The objective function of PCA is defined as below

$$
\begin{aligned}
J(U, C)= & \sum_{i=1}^{c} \sum_{j=1}^{n} u_{i j}^{m}\left\|x_{j}-c_{i}\right\|^{2} \\
& +\frac{\beta}{m^{2} \sqrt{c}} \sum_{i=1}^{c} \sum_{j=1}^{n}\left(u_{i j}^{m} \ln u_{i j}^{m}-u_{i j}^{m}\right) .
\end{aligned}
$$

where $x_{j}$ is the grey value of the $j$ th pixel, $c_{i}$ represents the center value of the $i$ th cluster, $n$ is the total number of pixels, $u_{i j}$ stands for the possibilistic membership of the $j$ th pixel with respect to cluster $i$, the weighting exponent $m \in(1,+\infty)$ is called the fuzzifier, $c$ denotes the cluster number and the sample co-variance $\beta$ is expressed as below

$$
\beta=\frac{1}{n} \sum_{j=1}^{n}\left\|x_{j}-\bar{x}\right\|^{2}, \quad \text { with } \quad \bar{x}=\frac{1}{n} \sum_{j=1}^{n} x_{j}
$$

It has been proved that the necessary conditions for a minimizer of the objective function in (1) are the following update equations

$$
u_{i j}=\exp \left(-\frac{m \sqrt{c}}{\beta}\left\|x_{j}-c_{i}\right\|^{2}\right), \quad \begin{aligned}
& i=1, \ldots, c \\
& j=1, \ldots, n
\end{aligned}
$$

and

$$
c_{i}=\frac{\sum_{j=1}^{n} u_{i j}^{m} x_{j}}{\sum_{j=1}^{n} u_{i j}^{m}}, \quad i=1, \ldots, c .
$$

A straightforward description of PCA_HS is this: In PCA HS, the histogram of the image is first obtained. Afterwards, PCA is applied on it. Finally, spatial information is used to revise isolated pixels. The following parts depict these steps in detail.

To explain the process of employing the grey-level histogram, let $k$ represent a certain grey value of the image and $h(k)$ denote the number of pixels with grey value $k$. For later use, let $L$ stand for the number of different grey values in the image. Now the distance of a pixel with grey value $k$ to the $i$ th cluster is below

$$
d_{i k}=\left\|k-c_{i}\right\|, \quad k \in\left\{k^{\prime} \mid h\left(k^{\prime}\right) \neq 0\right\} .
$$

The membership function in (3) turns to be

$$
t_{i k}=\exp \left(-\frac{m \sqrt{c}}{\beta}\left\|k-c_{i}\right\|^{2}\right), \quad \begin{gathered}
i=1, \ldots, c, \\
k \in\left\{k^{\prime} \mid h\left(k^{\prime}\right) \neq 0\right\} .
\end{gathered}
$$

Correspondingly, the center of a cluster in (4) becomes

$$
c_{i}=\frac{\sum_{k \in\left\{k^{\prime} \mid h\left(k^{\prime}\right) \neq 0\right\}} t_{i k}^{m} h(k) k}{\sum_{k \in\left\{k^{\prime} \mid h\left(k^{\prime}\right) \neq 0\right\}} t_{i k}^{m} h(k)} .
$$

The relationship between $u_{i j}$ and $t_{i k}$ is as follow

$$
u_{i j}=t_{i k}, \quad \text { if } \quad x_{j}=k
$$

Finally, the objective function is expressed as below

$$
\begin{aligned}
J(T, C)= & \sum_{i=1}^{c} \sum_{k \in\left\{k^{\prime} \mid h\left(k^{\prime}\right) \neq 0\right\}} t_{i k}^{m} d_{i k}^{2} h(k) \\
& +\frac{\beta}{m^{2} \sqrt{c}} \sum_{i=1}^{c} \sum_{k \in\left\{k^{\prime} \mid h\left(k^{\prime}\right) \neq 0\right\}}\left(t_{i k}^{m} \ln t_{i k}^{m}-t_{i k}^{m}\right) h(k) .
\end{aligned}
$$

The membership matrix $U$ of size $c \times n$ turns into $T$ of size $c \times L$. For a grey scale image of 8 bits, $L$ is no more than 256 , while $n$ is usually much bigger than 256 , and thus the grey-level histogram can help speed up the algorithm a lot.

Now that PCA has been applied on the histogram, each pixel in the image was divided into a cluster with a cluster number decided by the following equation

$$
m_{j}=i, \quad \max _{i} u_{i j}
$$

However, PCA with grey-level histogram is still sensitive to noise and outliers. For this reason, the spatial information is applied, and its main idea is that if hardly any neighbouring pixels are divided into the same cluster with the center pixel, it is reasonable to believe that this pixel is contaminated by noise and is wrongly divided. Therefore, we recursively reset its maximum membership 
function value to 0 by (11), and modify its cluster number by (12), until the new maximum value satisfies the second condition in (11). Finally adjust its grey value to the mean of its neighbouring pixels which are clustered to the same cluster with the center pixel.

Let $N$ represent the edge length of the pixels in the window. A $3 \times 3$ window except for the central pixel itself is used throughout this work. NP denotes the number of the neighbouring pixels that didn't divide into the same cluster with the center pixel, and thus the membership function can be defined as follow, and the initial value of $u_{i j}^{\prime}$ is $u_{i j}$ in (8).

$$
u_{i j}^{\prime}=\left\{\begin{array}{cc}
0, & N P /\left(N^{2}-1\right)>\varepsilon_{0}, i=m_{i j}^{\prime}, \\
u_{i j}^{\prime}, & N P /\left(N^{2}-1\right) \leq \varepsilon_{0}, i=m_{i j}^{\prime} .
\end{array}\right.
$$

The selection of $\varepsilon_{0}$ is analysed below. In Figure 1, it can be assumed that the center pixel in (a) and (b) are wrongly divided but correctly divided in (c). Values of $N P /\left(N^{2}-1\right)$ in Figure 1 are $1,0.875$ and 0.75 , respectively. Hence, it is rational to select $\varepsilon_{0} \in[0.75,0.875)$.

\begin{tabular}{|l|l|l|}
\hline 2 & 2 & 2 \\
\hline 3 & 1 & 3 \\
\hline 3 & 3 & 3 \\
\hline
\end{tabular}

(a)

\begin{tabular}{|l|l|l|}
\hline 1 & 2 & 2 \\
\hline 3 & 1 & 2 \\
\hline 3 & 3 & 3 \\
\hline
\end{tabular}

(b)

\begin{tabular}{|l|l|l|}
\hline 1 & $?$ & $?$ \\
\hline 1 & 1 & $?$ \\
\hline$?$ & $?$ & $?$ \\
\hline
\end{tabular}

(c)
Figure 1. a $3 \times 3$ window in the clustering domain. (a) Central pixel and its neighbors in different clusters. (b) One neighboring pixel in the same cluster with the central pixel. (c) More than one neighboring pixels in the same cluster with the central pixel.

In fact, this is a kind of filter technique in the cluster domain rather than image domain. Through this, we can effectively revise the wrongly divided pixels, while protecting the edge pixels between regions from being affected. Pixels in the image are finally divided by the revised cluster numbers defined as below

$$
m_{i j}^{\prime}=i, \quad \max _{i} u_{i j}^{\prime}
$$

The outline of PCA_HS is as below

Step 1: Decide the number of clusters; fix the fuzzifier $m$; set the iteration counter $l=1$; apply histogram for the grey-level image; estimate $\beta$ by (2);

Step 2: Find the initial fuzzy partition matrix $T$ by the standard FCM algorithm;

\section{Step 3: Repeat}

$$
\begin{aligned}
& \text { Compute } T^{(l+1)} \text { by (6); Increment } l \text {; } \\
& \text { Until }\left\|T^{(l-1)}-T^{(l)}\right\|<\varepsilon ;
\end{aligned}
$$

Step 4: Compute $u_{i j}^{\prime}$ by (8) and (11);

Step 5: Output the clustering result by (12) and then compute the validity indexes.

\subsection{Validity Indexes for PCA_HS}

The normalized possibilistic c-membership is defined as below

$$
\bar{u}_{i j}=\frac{u_{i j}^{\prime}}{\sum_{k=1}^{c} u_{k j}^{\prime}}, \quad \begin{aligned}
& i=1, \ldots, c \\
& j=1, \ldots, n .
\end{aligned}
$$

The generalized PC, PE, FS and XB defined in [10] can be sorted into two types, namely, the fuzzy partition type and the feature structure type [12]. GPC and GPE are the fuzzy partition type while GFS and GXB are the feature structure type. GPC and GPE are defined as below

$$
G P C=\frac{1}{n} \sum_{i=1}^{c} \sum_{j=1}^{n} \bar{u}_{i j}^{2}
$$

and

$$
G P E=-\frac{1}{n} \sum_{i=1}^{c} \sum_{j=1}^{n} \bar{u}_{i j} \ln \bar{u}_{i j} .
$$

The idea of GPC and GPE is that the partition with less fuzziness means better performance. As a result, the best clustering is achieved when GPC is maximized and GPE is minimized.

The above indexes use only the membership functions and lack a direct connection to the featuring properties. GFS and GXB are defined as below

$$
\begin{aligned}
G F S= & \sum_{i=1}^{c} \sum_{j=1}^{n} \bar{u}_{i j}{ }^{m}\left\|x_{j}-c_{i}\right\|^{2} \\
& -\sum_{i=1}^{c} \sum_{j=1}^{n} \bar{u}_{i j}^{m}\left\|c_{i}-\bar{c}\right\|^{2} .
\end{aligned}
$$

and

$$
G X B=\frac{\sum_{i=i}^{c} \sum_{j=1}^{n} \bar{u}_{i j}^{m}\left\|x_{j}-c_{i}\right\|^{2}}{n \min _{i \neq j}\left\|c_{i}-c_{j}\right\|^{2}} .
$$

where $\bar{c}$ is defined as below

$$
\bar{c}=\sum_{i=1}^{c} c_{i} / c .
$$

The idea of GFS and GXB is that the partition should be compact in clusters and separated from other clusters. The best clustering is achieved when GFS and GXB are minimized.

\section{Experiments and Discussions}

In this section, we conduct the experiment with and without Gaussian or Salt \& Pepper Noise on real images that are widely used by other researchers. The performance of PCA and PCA_HS are also compared. Throughout the experiment, the fuzzifier $m$ equals 1.5 , 
which is suggested in [8]. Two groups of the experimental results are presented as representatives.

\subsection{Results on Noise-Free Images}

Our first experiment applies PCA and PCA_HS on two noise-free images. The results are shown in Figure 2.

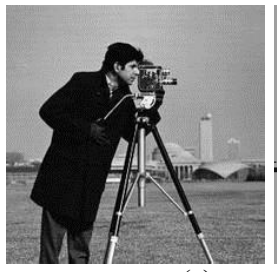

(a)

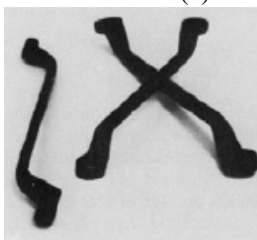

(d)

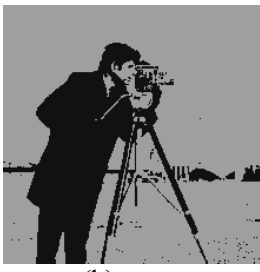

(b)

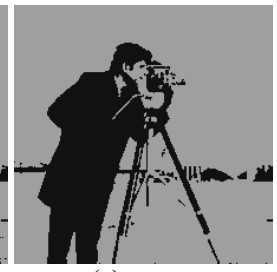

(c)

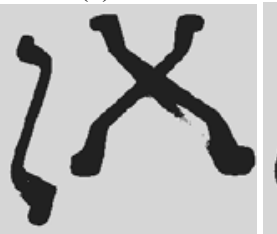

(e)

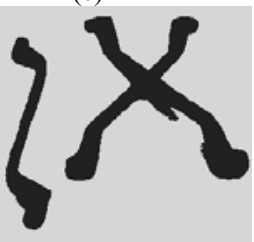

(f)
Figure 2. Segmentation results on noise-free images. First column: the original images; second column: PCA results of the corresponding images; third column: PCA_HS results of the corresponding images.

\subsection{Results on Gaussian Noised Images}

In this experiment, the original images are corrupted by zero-mean Gaussian Noise with its variance equal to 300 and the segmentation results are shown in Figure 3.

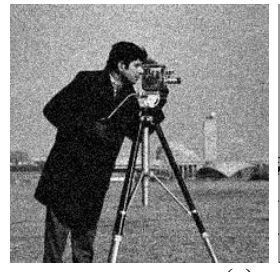

(a)

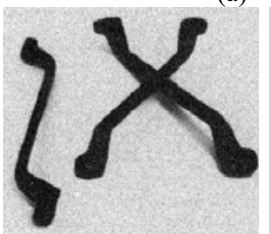

(d)

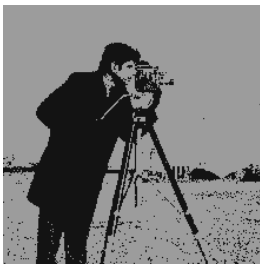

(b)

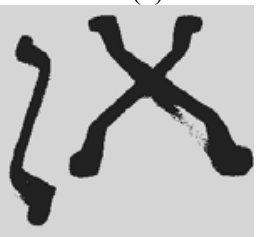

(e)

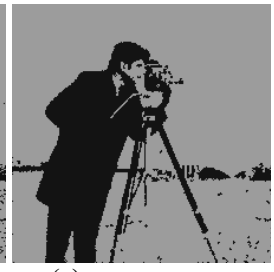

(c)

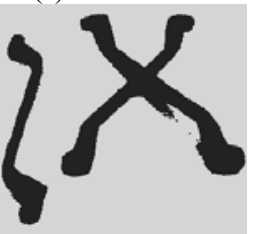

(f)
Figure 3. Segmentation results on zero-mean Gaussian noised images with variance equal to 300. First column: the noised images; second column: PCA results of the corresponding images; third column: PCA_HS results of the corresponding images.

\subsection{Results on Salt \& Pepper Noised Images}

In this experiment, the original images are corrupted by Salt \& Pepper Noise and the segmentation results are shown in Figure 4.

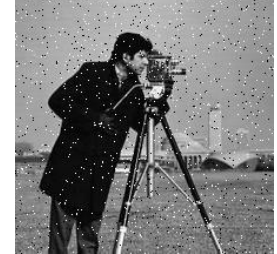

(a)
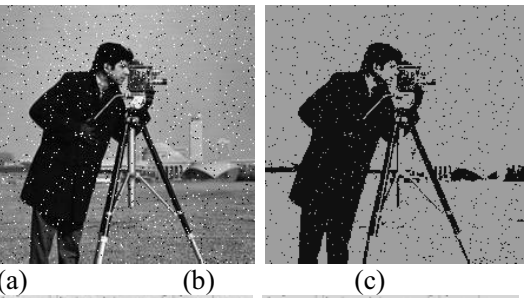

(c)

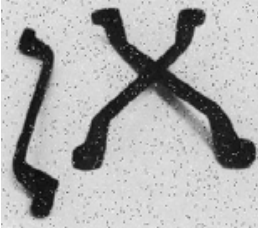

(e)

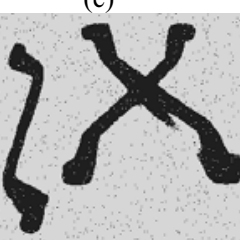

(f)

(d)
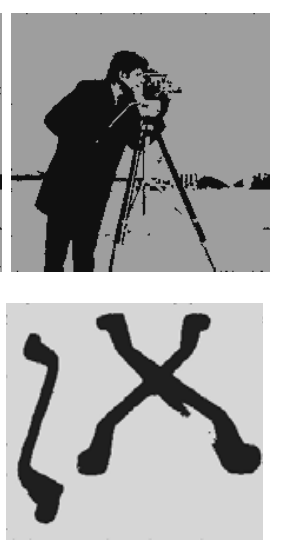

Figure 4. Segmentation results on Salt \& Pepper Noised images $(3 \%)$ First column: the noised images; second column: PCA results of the corresponding images; third column: PCA HS results of the corresponding images.

\subsection{Discussions}

As can be seen from Figure 2 to Figure 4, for both the cameraman and the spanner with and without noise, PCA retains many isolated pixels, while PCA_HS can accurately identify them and yield more homogeneous and integrated regions.

For more objective assessment, the validity indexes of the cameraman and the spanner are computed and the results are listed in Table I. As shown, for the cameraman and the spanner with and without noise, GPC of PCA_HS is bigger than PCA, and meanwhile GPE, GFS and GXB are all smaller than PCA. Other experiments reach similar results. This indicates that PCA HS is more effective than traditional PCA on real images with and without Gaussian or Salt \& Pepper Noise.

TABLE I. VALIDITY INDEXES FOR IMAGES WITH AND WITHOUT GaUsSian AND SAlt \& PePPER NoISE

\begin{tabular}{|l|l|l|l|l|l|}
\hline \multirow{2}{*}{ Images } & Algorithms & GPC & GPE & GXB & $\begin{array}{l}\text { GFS }(\times \\
\left.10^{8}\right)\end{array}$ \\
\hline \multirow{2}{*}{ Cameraman } & PCA & 0.9821 & 0.0322 & 0.02877 & -2.1120 \\
\cline { 2 - 6 } & PCA_HS & 0.9830 & 0.0307 & 0.02858 & -2.1163 \\
\hline \multirow{2}{*}{ Spanner } & PCA & 0.9955 & 0.0074 & 0.00620 & -5.7159 \\
\cline { 2 - 6 } & PCA_HS & 0.9957 & 0.0073 & 0.00618 & -5.7169 \\
\hline $\begin{array}{l}\text { Gaussian } \\
\text { Cameraman }\end{array}$ & PCA & 0.9725 & 0.0473 & 0.03482 & -1.8675 \\
\cline { 2 - 6 } & PCA_HS & 0.9762 & 0.0418 & 0.03200 & -1.8854 \\
\hline $\begin{array}{l}\text { Gaussian } \\
\text { Spanner }\end{array}$ & PCA & 0.9950 & 0.0086 & 0.00717 & -5.4754 \\
\cline { 2 - 6 } \begin{tabular}{l} 
Salt \& $\begin{array}{l}\text { Pepper HS } \\
\text { Cameraman }\end{array}$ \\
\cline { 2 - 6 }
\end{tabular} & PCA & 0.9952 & 0.0082 & 0.00681 & -5.4784 \\
\hline \begin{tabular}{l} 
Salt $\begin{array}{l}\text { Pepper } \\
\text { Spanner }\end{array}$ \\
\cline { 2 - 6 }
\end{tabular} & PCA & 0.9815 & 0.0337 & 0.03200 & -2.0785 \\
\cline { 2 - 6 } & PCA_HS & 0.9955 & 0.0076 & 0.00681 & -5.8220 \\
\hline
\end{tabular}

\section{Conclusions}

In this paper we proposed a possibilistic clustering algorithm incorporating the grey-level histogram and spatial information for image segmentation. Usually, the application of the grey-level histogram shrinks the membership matrix and thus can reduce the spatial and computational complexity. The spatial information is used for filtering in the cluster domain rather than image 
domain, and hence can enhance the robustness and meanwhile protect the edge pixels between regions from being affected. The efficiency analysis and the experiment results on real images with and without Gaussian or Salt \& Pepper Noise indicate that PCA_HS is faster and more robust than traditional PCA for image segmentation.

\section{Acknowledgment}

The authors would like to thank the anonymous reviewers for their valuable comments and helpful suggestions in improving the presentation of the paper. This work was supported in part by the Fundamental Research Funds for the Central Universities.

\section{References}

1. M.O. Baradez, C.P. McGuckinb, N. Forrazb, R. Pettengell, and A. Hoppe, "Robust and automated unimodal histogram thresholding and potential applications," Pattern Recognit., vol. 37, no. 6, pp. 1131-1148, 2004.

2. S.A. Hojjatoleslami, and J. Kittler, "Region growing: a new approach," IEEE Trans. Image Process., vol. 7, no. 7, pp. 1079-1084, 1998.

3. B. Caldairoua, N. Passata, P.A. Habas, C. Studholme, and F. Rousseau, "A non-local fuzzy segmentation method: application to brain MRI, ${ }^{\prime}$ Pattern Recognit., vol. 44 no.9, pp. 1916-1927, 2010.

4. B. Sowmya, and B.S. Rani, "Color image segmentation using fuzzy clustering techniques and competitive neural network," Appl. Soft Comput., vol.11 no.3, pp. 3170-3178, 2011.

5. W.L. Cai, S.C. Chen, and D.Q. Zhang, "Fast and robust fuzzy c-means clustering algorithms incorporating ", local information for image segmentation," Pattern Recognit., vol.40 no.3, pp. 825-838, 2007.

6. M. G. Gong, Y. Liang, J. Shi, W.P. Ma, and J.J. Ma, "Fuzzy C-Means Clustering With Local Information and Kernel Metric for Image Segmentation," IEEE Trans. Image Process., vol. 22, no. 2, pp. 573-584, Feb. 2013.

7. R. Krishnapuram, J.M. Keller, "A possibilistic approach to clustering," IEEE Trans. Fuzzy Syst., vol.1, no. 2, pp. 98-110, May 1993.

8. R. Krishnapuram, J.M. Keller, "The possibilistic Cmeans algorithm: insights and recommendations," IEEE Trans. Fuzzy Syst., vol.4, no.3, pp. 385-393, Aug 1996.

9. M. Barni, V. Cappellini, and A. Mecocci, "Comments on "A Possibilistic Approach to Clustering," IEEE Trans. Fuzzy Syst., vol. 4, pp. 393-396, Aug. 1996.

10. M. S. Yang, K. L. Wu, "Unsupervised Possibilistic Clustering," Pattern Recognit., vol.39, no.1, pp. 5-21, 2006.

11. Y.F. Xu, "Image Segmentation Based on the Genetic Fuzzy C-Mean Algorithm," Journal of Northwestern Polytechnical University, vol.20, no.4, pp. 549-553, November, 2002.

12. K.S. Chuang, H.L. Tzeng, S. Chen, J. Wu, and T.J. Chen, "Fuzzy c-means clustering with spatial information for image segmentation," Computerized Medical Imaging and Graphics, vol. 30 , no. 1, pp. 9$15,2006$.

13. H. Zuo, and W. Li, "Improved PCM Clustering Algorithm and Its Application in Image Segmentation," Computer \& Digital Engineering, vol.38, no.11, pp.148-151, June 2010. 